\title{
Mutual events of Jupiter's satellites observed in 1997 at the Bordeaux Observatory
}

\author{
G. Dourneau ${ }^{1}$, J. F. Le Campion ${ }^{1}$, and S. Baratchart ${ }^{2}$ \\ 1 Observatoire Aquitain des Sciences de l'Univers, UMR 5804, Laboratoire d'Astrodynamique, d'Astrophysique \\ et d'Aéronomie de Bordeaux (L3AB), 2 rue de l'Observatoire, 33270 Floirac, France \\ e-mail: dourneau@obs.u-bordeaux1.fr \\ 2 Observatoire Midi Pyrénées, 14 avenue Edouard Belin, 31400 Toulouse, France
}

\section{Received 2 July 2004 / Accepted 21 February 2005}

\begin{abstract}
We present photometric observations of mutual events of the Galilean satellites of Jupiter made in 1997 at the Bordeaux Observatory as part of the international PHEMU97 campaign. We observed 21 events which provided 14 high quality light curves with the CCD camera mounted on the $62 \mathrm{~cm}$ telescope. From these curves, we determined observed values and errors for the maximum intensity drop and the time of this light minimum. Phase correction was applied to this time for a proper comparison to theoretical values. Residuals between observed and computed values of both of these parameters have been derived from Arlot's G5 ephemerides. The discussion of errors and residuals shows that these observational parameters generally are so accurately determined that they lead to satellite positions with an angular precision of about ten milliarcseconds, never obtained with classical astrometry. In addition, some theoretical results have been derived from the analysis of our set of observations. The main result concerns the satellite J1 for which our observations of the time of maximum event suggest an advance in longitude of $269 \mathrm{~km}$ with respect to G5. Also, for the satellite J4, we similarly obtain a delay of about $170 \mathrm{~km}$ which appears to be a real effect.
\end{abstract}

Key words. eclipses - occultations - planets and satellites: individual: Galilean satellites

\section{Introduction}

The Bordeaux Observatory has been involved in studies of satellite dynamics for decades. The observatory has participated in previous international campaigns of the observation of mutual events involving the satellites of the giant planets Jupiter and Saturn (Arlot et al. 1982; Dourneau 1982; Arlot et al. 1992). Initially, a photometer was used. In the nineties, the photometer was replaced by a CCD camera which appeared quite well adapted to observe mutual events, as shown during the PHEMU91 and PHESAT95 campaigns in 1991 and 1995, respectively (Le Campion et al. 1992; Arlot et al. 1997; Thuillot et al. 2001). We used this CCD camera for the PHEMU97 campaign and our results are presented in this paper. First, we describe our observational methods and present the 21 observed events. Then, the reduction of observed raw data is explained which leads to observed values and errors of observational parameters for each event. We then compare our observed values of these parameters to those predicted by Arlot (1996) from his G5 ephemerides (Arlot 1982). The obtained residuals are discussed and we derive some theoretical results.

\section{Observations}

The PHEMU97 campaign of observations of mutual events of the Galilean satellites at the Bordeaux Observatory observed the 21 events listed in Table 1.
Our CCD camera, mounted on the $62 \mathrm{~cm}$ telescope of the Bordeaux Observatory, is equipped with a Thomson TH7863 chip of $384 \times 288$ pixels of $23 \times 23 \mu \mathrm{m}$. As the focal length of the telescope is close to $10 \mathrm{~m}$, each pixel corresponds to about 0.5 arcsec and the field on the CCD chip is about $3^{\prime} \times 2^{\prime}$. Our acquisition program allows us to save images within windows including only the occulted or eclipsed satellite and, when possible, another satellite used as a reference. As these windows are much smaller than the whole matrix, we obtain a significant reduction of the acquisition time, and consequently a higher frequency of images. This frequency is of about 1 image every $3 \mathrm{~s}$ with our camera. Dark counts and flat fields were performed each night of observation, except for 24/04/1997 and 06/06/1997, so that proper photometric corrections could be made for thermal background and for the variation of pixel sensitivity. Sky background was modeled with a polynomial function and removed from the observed image (Le Campion et al. 1992).

We also observed events about $10 \mathrm{~min}$ before the predicted start time, as well as $10 \mathrm{~min}$ after the predicted end. We thus obtain before and after each event a flat light level of the intensity that is long enough to provide an accurate reference level. This will lead to a better determination of the observed maximum intensity drop $I D_{\mathrm{M}}$ of the event. 
Table 1. List of all mutual events observed at the Bordeaux Observatory during the PHEMU97 campaign. Type of events are occulations (O) or eclipses (E). They can be total (T), partial (P) or annular (A). Predicted values of maximum intensity drop are from Arlot's G5 ephemerides. Reference satellites, when available, and integration time for each image of the sequence are mentioned, as well as the number of images and the duration of the sequence of each event.

\begin{tabular}{|c|c|c|c|c|c|c|c|}
\hline $\begin{array}{l}\text { Date of } \\
\text { observation }\end{array}$ & $\begin{array}{l}\text { Type of } \\
\text { event }\end{array}$ & $\begin{array}{l}\text { Predicted } \\
\text { maximum } \\
\text { intensity drop }\end{array}$ & $\begin{array}{l}\text { Reference } \\
\text { satellite }\end{array}$ & $\begin{array}{l}\text { Integration } \\
\text { time (s) }\end{array}$ & $\begin{array}{l}\text { Number } \\
\text { of } \\
\text { images }\end{array}$ & $\begin{array}{l}\text { Sequence } \\
\text { duration } \\
\text { (s) }\end{array}$ & Observers $^{a}$ \\
\hline $24 / 04 / 1997$ & $4 \mathrm{O} 2 \mathrm{~T}$ & 0.295 & S3 & 2.0 & 166 & 394 & JL/GD \\
\hline 30/05/1997 & $301 \mathrm{~T}$ & 0.321 & S2 & 2.0 & 220 & 603 & $\mathrm{JL} / \mathrm{GD}$ \\
\hline 06/06/1997 & $3 \mathrm{O} 2 \mathrm{P}$ & 0.176 & $\mathrm{~S} 1+\mathrm{S} 4$ & 3.0 & 126 & 437 & $\mathrm{GD} / \mathrm{SB}$ \\
\hline 18/06/1997 & $2 \mathrm{E} 1 \mathrm{~A}$ & 0.647 & None & 0.2 & 169 & 298 & $\mathrm{GD} / \mathrm{SB}$ \\
\hline 25/06/1997 & $2 \mathrm{E} 1 \mathrm{~A}$ & 0.640 & None & 10.0 & 63 & 294 & $\mathrm{JL} / \mathrm{GB} / \mathrm{SB}$ \\
\hline 07/07/1997 & 1E2 & 0.116 & S1 & 0.5 & 252 & 316 & GD/SB \\
\hline 09/07/1997 & $2 \mathrm{E} 3$ & 0.095 & $\mathrm{~S} 2$ & 0.2 & 233 & 398 & $\mathrm{GD} / \mathrm{SB}$ \\
\hline 16/07/1997 & $2 \mathrm{E} 3 \mathrm{~A}$ & 0.208 & $\mathrm{~S} 2$ & 0.5 & 265 & 440 & $\mathrm{GD} / \mathrm{SB}$ \\
\hline 19/07/1997 & $2 \mathrm{E} 1$ & 0.149 & S3 & 0.5 & 253 & 233 & GD \\
\hline 27/07/1997 & $2 \mathrm{E} 1$ & 0.050 & S3 & 3.0 & 77 & 194 & GD \\
\hline 29/07/1997 & $103 \mathrm{P}$ & 0.206 & None & 0.3 & 342 & 346 & SB \\
\hline 22/08/1997 & $1 \mathrm{E} 2$ & 0.004 & S1 & 1.0 & 363 & 857 & $\mathrm{JL} / \mathrm{GD}$ \\
\hline 11/09/1997 & 103 & 0.204 & $\mathrm{~S} 2$ & 0.1 & 548 & 1323 & $\mathrm{JL} / \mathrm{GD}$ \\
\hline 18/09/1997 & $1 \mathrm{E} 3 \mathrm{~A}$ & 0.472 & S1 & 0.5 & 545 & 561 & $\mathrm{JL} / \mathrm{GD}$ \\
\hline 19/09/1997 & $403 \mathrm{P}$ & 0.061 & None & 0.5 & 603 & 784 & GD \\
\hline 22/09/1997 & $3 \mathrm{E} 2 \mathrm{P}$ & 0.673 & $\mathrm{~S} 3+\mathrm{S} 4$ & 1.5 & 440 & 902 & $\mathrm{JL} / \mathrm{GD}$ \\
\hline $25 / 09 / 1997$ & $1 \mathrm{E} 3 \mathrm{~A}$ & 0.449 & S1 & 0.5 & 434 & 506 & $\mathrm{JL} / \mathrm{GD}$ \\
\hline 28/09/1997 & $301 P$ & 0.163 & None & 1.0 & 325 & 274 & $\mathrm{JL} / \mathrm{GD}$ \\
\hline 28/09/1997 & 3E1P & 0.483 & $\mathrm{~S} 2$ & 1.0 & 231 & 402 & $\mathrm{JL} / \mathrm{GD}$ \\
\hline 29/09/1997 & $3 \mathrm{E} 2 \mathrm{P}$ & 0.229 & $\mathrm{~S} 1+\mathrm{S} 3$ & 1.0 & 287 & 685 & $\mathrm{JL} / \mathrm{GD}$ \\
\hline 02/10/1997 & $1 \mathrm{O} 3 \mathrm{P}$ & 0.041 & None & 1.0 & 339 & 243 & GD \\
\hline
\end{tabular}

a JL: J.F. Le Campion, GD: G. Dourneau, SB: S. Baratchart.

\section{Measurement and reduction}

We developed a custom program called Phemufit to measure CCD images and to reduce observed raw data obtained from mutual events. This program performs four successive steps.

\subsection{Identification}

The first step consists of creating an identification file to be associated with the sequence of images saved during each observed event. This file records the type of each event, as given in Col. 2 of Table 1. For example, $4 \mathrm{O} 2$ means that $\mathrm{J} 4$ occults $\mathrm{J} 2$; $2 \mathrm{E} 1$ means that $\mathrm{J} 2$ eclipses $\mathrm{J} 1$; the following letters $\mathrm{T}, \mathrm{P}$ or $\mathrm{A}$ mean total, partial or annular event. Also mentioned in the identification file of each event are the day, month and year of observation, the integration time of each image and the number of images in the sequence. Most of the observational information included in the identification files is given in Table 1 for each of the 21 observed events.

\subsection{Measurement}

Our program Phemufit allows an automatic measurement of the same satellite present on each image of a sequence. This automatic procedure is possible as satellites move very slowly during an event. Mutual events need a photometric measurement of the light intensity $I$ of satellites whose variations have to be determined. Then, for each sequence, the images of each observed satellite are framed within a small window of $17 \times 17$ pixels. As this satellite stays within this window for the whole sequence, its light intensity $I$ can be measured in such a window along the sequence automatically.

\subsection{Analysis}

The third step of analysis consists of obtaining the raw light curve of events which gives, as a dot for each image of the sequence, the variable intensity $I$ of the eclipsed or occulted satellite versus time. When a reference satellite is available, the light curve displays the ratio of the satellite variable intensity over the constant intensity of the reference satellite. This procedure presents the advantage of reducing possible disturbing intensity variations, independent of the event, which can occur for example with a cloudy sky. Using a reference satellite allows us to obtain raw light curves with a lower dispersion of dots as a result of an improved signal to noise ratio of the intensity $I$ of the observed satellites. Figure 1 presents the light curves of the 14 events for which the variation of satellite light intensity $I$ has been detected.

\subsection{Determination of observational parameters}

During the fourth step we fit the raw light curve of each event. The central part of this curve is fitted to a polynomial model of the second order so as to obtain the first observational parameter $T_{\mathrm{Ml}}$ which is the time of light minimum. Both extremities of 

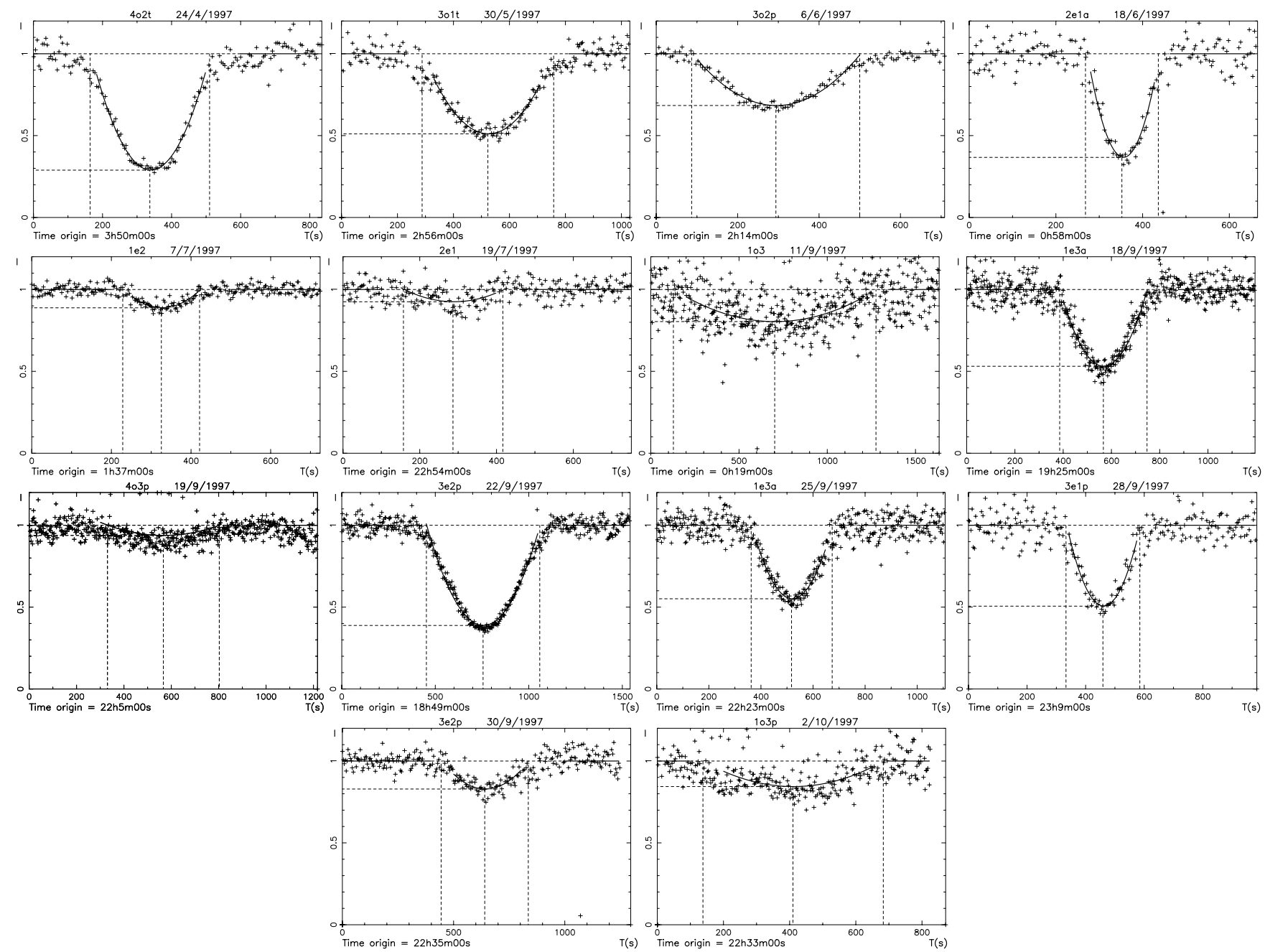

Fig. 1. Light curves of the 14 detected mutual events observed at the Bordeaux Observatory during the PHEMU97 campaign. The intensity $I$ of the eclipsed or occulted satellite is linearly fitted out of event and polynomially fitted during event. The flat level of satellite intensity out of event is normalized to the arbitrary value $I=1$. Time argument $T$ is expressed in seconds of Universal Time (UT).

the curve, obtained about 10 min before and after the event, are fitted together to a linear model in order to obtain the reference out of event intensity level. The value of this reference level is arbitrarily normalized to $I=1$ so as to determine more easily the maximum intensity drop $I D_{\mathrm{M}}$ which is the second observational parameter. Figure 1 displays such linear and polynomial fitted sections of light curves for the 14 detected mutual events. For both of these parameters, observational errors are derived from their respective least square polynomial fits.

\section{Results and discussion}

\subsection{Results of observations}

The results of our observations are presented in Table 2 giving the observed values and errors of both observational parameters $I D_{\mathrm{M}}$ and $T_{\mathrm{Ml}}$ for the 14 events that have been successfully detected. $T_{\mathrm{Ml}}$ is expressed in Universal Time (UT). As mentioned in Sect. 2, no proper photometric corrections could be made for the 2 events 4O2T (24/04/1997) and 3O2P (06/06/1997). Consequently, the corresponding observed values of $I D_{\mathrm{M}}$ may not be properly calibrated.
When comparing Table 2 with Table 1, we can see that 7 events among the 21 observed ones listed in Table 1 do not appear in Table 2. For these 7 events, the variation of the intensity of occulted or eclipsed satellites has not been detected. This can be because some of these events were too faint to be detected as 1E2 (22/08/1997) with a predicted maximum intensity drop $I D_{\mathrm{M}}$ of only 0.004 . For other events, this should be a consequence of the absence of a reference satellite, as for events 2E1A (25/06/1997), 1O3P (29/07/1997) and $301 P(28 / 09 / 1997)$. Without any reference satellite, the signal to noise ratio decreases and the light curves present a high dispersion of dots. For the 3 other undetected events, satellites were situated too close to the bright planet Jupiter, at a critical distance of 1.5 Jupiter radii, which results in a low signal to noise ratio. The observation of these 7 events have thus resulted in either flat or noisy light curves from which no determination of observational parameters was possible.

On the other hand, the event 2E1 (19/07/1997) which seemed undetectable from a single visual observation of the raw light curve (Fig. 1) was successfully determined from the polynomial fit. Some events without a reference satellite were 
Table 2. Observed values and errors of observational parameters $I D_{\mathrm{M}}$ and $T_{\mathrm{Ml}}$ for the 14 detected events. $I D_{\mathrm{M}}$ is the maximum intensity drop and $T_{\mathrm{Ml}}$ is the time of light minimum. $\Delta \mathrm{t}$ is the time shift due to the phase effect (Aksnes et al. 1986), between the time of light minimum and the time of geometric closest approach of satellites centers. $T_{\mathrm{Ml}}$ is expressed in Universal Time (UT).

\begin{tabular}{ccccc}
\hline \hline Date & Type of event & $I D_{\mathrm{M}}$ & $T_{\mathrm{Ml}}$ & $\Delta t(\mathrm{~s})$ \\
\hline $24 / 04 / 1997$ & 4O2T & $0.711^{a} \pm 0.038$ & $03 \mathrm{~h} 55 \mathrm{~m} 50 \mathrm{~s} \pm 0 \mathrm{~m} 16 \mathrm{~s}$ & +6 \\
$30 / 05 / 1997$ & 3O1T & $0.489 \pm 0.037$ & $03 \mathrm{~h} 05 \mathrm{~m} 06 \mathrm{~s} \pm 0 \mathrm{~m} 37 \mathrm{~s}$ & +9 \\
$06 / 06 / 1997$ & 3O2P & $0.316^{a} \pm 0.036$ & $02 \mathrm{~h} 19 \mathrm{~m} 06 \mathrm{~s} \pm 0 \mathrm{~m} 22 \mathrm{~s}$ & +7 \\
$18 / 06 / 1997$ & 2E1A & $0.633 \pm 0.170$ & $01 \mathrm{~h} 04 \mathrm{~m} 40 \mathrm{~s} \pm 0 \mathrm{~m} 44 \mathrm{~s}$ & -4 \\
$07 / 07 / 1997$ & $1 \mathrm{E} 2$ & $0.112 \pm 0.030$ & $01 \mathrm{~h} 42 \mathrm{~m} 29 \mathrm{~s} \pm 1 \mathrm{~m} 25 \mathrm{~s}$ & -3 \\
$19 / 07 / 1997$ & 2E1 & $0.075 \pm 0.068$ & $22 \mathrm{~h} 59 \mathrm{~m} 18 \mathrm{~s} \pm 3 \mathrm{~m} 00 \mathrm{~s}$ & -1 \\
$11 / 09 / 1997$ & $1 \mathrm{O} 3$ & $0.196 \pm 0.158$ & $00 \mathrm{~h} 31 \mathrm{~m} 34 \mathrm{~s} \pm 3 \mathrm{~m} 43 \mathrm{~s}$ & +19 \\
$18 / 09 / 1997$ & 1E3A & $0.469 \pm 0.056$ & $19 \mathrm{~h} 34 \mathrm{~m} 36 \mathrm{~s} \pm 0 \mathrm{~m} 37 \mathrm{~s}$ & +7 \\
$19 / 09 / 1997$ & 4O3P & $0.062 \pm 1.006$ & $22 \mathrm{~h} 15 \mathrm{~m} 22 \mathrm{~s} \pm 6 \mathrm{~m} 22 \mathrm{~s}$ & -13 \\
$22 / 09 / 1997$ & 3E2P & $0.612 \pm 0.034$ & $19 \mathrm{~h} 01 \mathrm{~m} 56 \mathrm{~s} \pm 0 \mathrm{~m} 21 \mathrm{~s}$ & +9 \\
$25 / 09 / 1997$ & 1E3A & $0.449 \pm 0.044$ & $22 \mathrm{~h} 32 \mathrm{~m} 19 \mathrm{~s} \pm 0 \mathrm{~m} 42 \mathrm{~s}$ & +7 \\
$28 / 09 / 1997$ & 3E1P & $0.494 \pm 0.056$ & $23 \mathrm{~h} 17 \mathrm{~m} 15 \mathrm{~s} \pm 0 \mathrm{~m} 50 \mathrm{~s}$ & +4 \\
$29 / 09 / 1997$ & 3E2P & $0.171 \pm 0.051$ & $22 \mathrm{~h} 46 \mathrm{~m} 19 \mathrm{~s} \pm 3 \mathrm{~m} 41 \mathrm{~s}$ & +7 \\
$02 / 10 / 1997$ & 1O3P & $0.156 \pm 2.043$ & $22 \mathrm{~h} 39 \mathrm{~m} 56 \mathrm{~s} \pm 2 \mathrm{~m} 59 \mathrm{~s}$ & -5 \\
\hline
\end{tabular}

${ }^{a}$ Unreliable intensity calibration.

detected: 2E1A (18/06/1997), 4O3P (19/09/1997) and 1O3P (2/10/1997). For 2E1A, this positive result can be explained as it presents one of the largest maximum intensity drops $I D_{\mathrm{M}}$ of the observed events as well as one of the shortest durations. For 4O3P and 1O3P, presenting rather low maximum intensity drops and long durations, the detection was possible but the events were very faint. Consequently, they present some of the highest observational errors of Table 2 . This illustrates the utility of the reference satellite in observing mutual events.

For all other events, Table 2 shows that the maximum intensity drop $I D_{\mathrm{M}}$ is accurately determined with an average error of a few percent. Also, the time of light minimum $T_{\mathrm{Ml}}$ is quite well determined from observations, with errors generally less than half a minute. These errors correspond to satellite positions of a few tens of milliarcseconds, significantly lower than those generally obtained from classical astrometry.

\subsection{Comparison with theory}

We now compare observed values of both observational parameters given in Table 2 to predicted values by Arlot (1996) from his G5 ephemerides (Arlot 1982). The observed values of $T_{\mathrm{Ml}}$ are expressed in Universal Time (UT) while the corresponding predicted times by Arlot were given in Ephemeris Time (ET). For a proper computation of these time residuals, we have used the differences $\Delta T=\mathrm{ET}-\mathrm{UT}$ available for the year 1997 and given in the Connaissances des Temps for 1999. These values are $\Delta T=62.184 \mathrm{~s}$ before $1 / 07$ and $\Delta T=63.184 \mathrm{~s}$ after $1 / 07$.

The parameter $T_{\mathrm{Ml}}$ as determined above corresponds to the time of light minimum. At this time, the light center of the occulted or eclipsed satellite is closest to the geometric center of the occulting satellite or to the shadow center of the eclipsing satellite. For a proper comparison to the theory, we have evaluated the time shift $\Delta t$ between the light center and the geometric center of the occulted or eclipsed satellite due to the phase effect (Aksnes et al. 1986) for each event, as given in Table 2. This shift must be added to $T_{\mathrm{Ml}}$ so as to obtain $T_{\mathrm{Mg}}$, the time of midevent, which corresponds to the observed time of closest approach of the geometric centers of the satellites. $T_{\mathrm{Mg}}$ is now comparable to the predicted times of midevents derived from theory. We thus obtain the residuals $(\mathrm{O}-\mathrm{C})$ presented in Table 3.

When analysing Table 3, we can see that residuals for the maximum intensity drop $I D_{\mathrm{M}}$ are very low; for 10 out of 14 events these are only a few percent and for 5 of the events less than 1 percent. As $I D_{\mathrm{M}}$ is strongly linked to satellite latitude, low values of the residuals for this parameter correspond to a very high accuracy of the satellite position in latitude; the residuals are as low as about one milliarsecond. Also, the residuals for the time of midevent $T_{\mathrm{Mg}}$, related to the longitude of the satellites, are very low. We can see that 8 of them are under $10 \mathrm{~s}$, corresponding to an accuracy of a few tens of milliarcseconds in satellite longitude positions. All other residuals in $T_{\mathrm{Mg}}$ remain lower than $37 \mathrm{~s}$, also corresponding to a good accuracy, significantly better than that derived from most classical astrometric satellite observations.

For a more realistic interpretation of the analysis of residuals, we have converted all residuals in $T_{\mathrm{Mg}}$ from timings to distances by multiplying them by the relative sky plane velocity of satellites, given for each event on the Web site of the Institut de Mécanique Céleste et de Calcul des Ephémérides (IMCCE). The derived values are presented in Table 3 and will be discussed in the following section.

\subsection{Discussion}

When comparing observational errors of both intensity and time parameters given in Table 2 with their corresponding residuals of Table 3, we can see that residuals generally are significantly lower than their respective errors. This suggests that our method of evaluation of observational errors of parameters, directly derived from least squares solutions, should have overestimated these errors. This can enhance the accuracy of our observations of mutual events. 
Table 3. Residuals (O-C) of observational parameters $I D_{\mathrm{M}}$ and $T_{\mathrm{Mg}}$ derived from the comparison of the mutual events observed in 1997 at the Bordeaux Observatory to G5. Time residuals are given in seconds with their equivalent residuals in distance, expressed in $\mathrm{km}$.

\begin{tabular}{ccccc}
\hline \hline $\begin{array}{c}\text { Date of } \\
\text { observation }\end{array}$ & $\begin{array}{c}\text { Type of } \\
\text { event }\end{array}$ & $I D_{\mathrm{M}}$ & $\begin{array}{c}\text { Residuals (O-C) } \\
T_{\mathrm{Mg}}(\mathrm{sec})\end{array}$ & Distance $(\mathrm{km})$ \\
\hline $24 / 04 / 1997$ & $4 \mathrm{O} 2 \mathrm{~T}$ & +0.416 & +8 & +161 \\
$30 / 05 / 1997$ & $3 \mathrm{O} 1 \mathrm{~T}$ & $+0.168(0.043)^{a}$ & -3 & -44 \\
$06 / 06 / 1997$ & $3 \mathrm{O} \mathrm{P}$ & +0.140 & +24 & +402 \\
$18 / 06 / 1997$ & $2 \mathrm{E} 1 \mathrm{~A}$ & -0.014 & -8 & -230 \\
$07 / 07 / 1997$ & $1 \mathrm{E} 2$ & -0.004 & +12 & +226 \\
$19 / 07 / 1997$ & $2 \mathrm{E} 1$ & -0.074 & -37 & -1127 \\
$11 / 09 / 1997$ & $1 \mathrm{O} 3$ & -0.008 & -35 & -209 \\
$18 / 09 / 1997$ & $1 \mathrm{E} 3 \mathrm{~A}$ & -0.003 & -9 & -179 \\
$19 / 09 / 1997$ & $4 \mathrm{O} \mathrm{P}$ & +0.001 & +22 & +178 \\
$22 / 09 / 1997$ & $3 \mathrm{E} 2 \mathrm{P}$ & -0.061 & +9 & +92 \\
$25 / 09 / 1997$ & $1 \mathrm{E} 3 \mathrm{~A}$ & 0.000 & -9 & -199 \\
$28 / 09 / 1997$ & 3E1P & +0.011 & -7 & -181 \\
$29 / 09 / 1997$ & $3 \mathrm{E} 2 \mathrm{P}$ & -0.058 & +2 & +23 \\
$02 / 10 / 1997$ & $1 \mathrm{O} 3 \mathrm{P}$ & +0.115 & -23 & -477 \\
\hline
\end{tabular}

${ }^{a}$ Comparison to recent model by Descamps (2004).

The first three events listed in Table 3, 4O2T (24/04/1997), $3 \mathrm{O} 1 \mathrm{~T}(30 / 05 / 1997)$ and 3O2P (6/06/1997), all present significative positive residuals in $I D_{\mathrm{M}}$, higher than 0.1 . Simultaneously, the corresponding observational errors are lower, showing the quite good determination of this parameter which should then lead to some valuable latitude corrections of satellites. But, as we have already mentioned in Sect. 4.1 these 2 events, 4O2T (24/04/1997) and 3O2P (06/06/1997), present an intensity which may not be reliable. Their corresponding residuals in $I D_{\mathrm{M}}$ should not be taken into account for any later theoretical corrections. On the other hand, for the last of these three events, 3O1T (30/05/1997), the photometric corrections were performed properly. We have compared our observed parameter $I D_{\mathrm{M}}$ to a value derived from a sophisticated model including albedo variations on satellite surfaces, recently proposed by Descamps (2004) for this peculiar event. The new residual dropped to a value of 0.043 , near the accuracy of the observations.

As another example of a possible correction of the theory derived from our observations, we can analyse residuals in $T_{\mathrm{Mg}}$ given in Table 3 . Most of these residuals are negative. To rely on satellite longitude residuals, we must consider the geometry of satellites during the observed events, as given in the Annuaire du Bureau des Longitudes for 1997. For all events, except one, the occulting or eclipsing satellite is moving from east to west when the other satellite is moving from west to east. The exception is for the longer event $103(11 / 09 / 1997)$ where both satellites are moving from west to east. From the residuals of Table 3, we can derive the approximate corrections to the longitude of the satellites involved. Unexpectedly, for satellite $\mathrm{J1}$, we can see that all residuals are negative, except one, for the event 1E2 (7/07/1997). When computing the average value of residuals in $T_{\mathrm{Mg}}$ for the 9 events involving $\mathrm{J} 1$, we obtain $-13 \mathrm{~s}$. For these 9 events, we obtain an equivalent mean residual in distance of $-269 \mathrm{~km}$ for $\mathrm{J} 1$ with respect to G5. This mean residual also can affect satellites J2 and J3 with which $\mathrm{J} 1$ was involved in these 9 events. However, satellite $\mathrm{J} 1$ should be the most affected by this residual as it is involved in all of the 9 events, while $\mathrm{J} 2$ is involved in only 3 of them and $\mathrm{J} 3$ in 6 of them. This suggests that the theoretical longitude of $\mathrm{J} 1$ may need a possible correction of $269 \mathrm{~km}$, in advance with respect to G5. Similar results were previously obtained from the comparison of the mutual events observed in 1991 (Mallama 1992; Vasundhara 1994) with respect to Lieske's theory E3, an updated version of his original theory and previous sets of constants E1 and E2 (Lieske 1977, 1978, 1980).

For other satellites, results derived from a similar computation of average residuals do not lead to values as high as for J1. For $\mathrm{J} 2$, the mean residual only reaches the value of $-65 \mathrm{~km}$ for 7 observed events, and for $\mathrm{J} 3$ we have obtained a value of $-59 \mathrm{~km}$ for 10 observed events involving this satellite. So, it seems very difficult to suggest any correction in longitude from our observations for both $\mathrm{J} 2$ and $\mathrm{J} 3$, as their mean residuals are as low as the accuracy of observations. For satellite J4, we obtain a significant positive mean time residual of $+15 \mathrm{~s}$ for 2 observed events, corresponding to a delay of $170 \mathrm{~km}$ in the longitude of this satellite with respect to G5. Such a delay was also noticed by Vasundhara et al. (2003) with respect to Lieske's theory E3 and could be a real effect.

\section{Conclusion}

This work emphasizes the high quality of mutual event observations for deriving accurate planetary satellite positions. We have seen that the maximum intensity drop and the time of midevent were quite accurately determined from our observations. The observational errors of these parameters correspond to satellite positions with an accuracy of the order of ten milliarcseconds, significantly better than the accuracy of classical astrometric satellite observations. Moreover, for both of these parameters, we obtain residuals that are generally lower than the observational errors. This suggests that our errors have been over-evaluated, and consequently that our observations are actually more accurate. 
Second, we mention some theoretical results derived from the analysis of observational parameter residuals. The main result concerns the longitude of satellite J1. For this satellite, the observed times of midevent, strongly related to satellite longitude, suggest a possible correction of its longitude by $269 \mathrm{~km}$, in advance with respect to G5. Similar results had been obtained by Mallama (1992) and Vasundhara (1994) when comparing the mutual events of 1991 to Lieske's theory E3. In addition, the analysis of our observations has led to another interesting result in the determination of a delay of $170 \mathrm{~km}$ in the longitude of $\mathrm{J} 4$ with respect to $\mathrm{G} 5$, which appears to be a real effect.

The results obtained in this paper about a possible correction of the orbital longitude or latitude of the Galilean satellites are not only interesting in themselves, but also because they show the strength of mutual event observations for deriving theoretical information. It would be useful to improve the accuracy of these mutual event observations so as to be able to improve theories of satellite motion. This could be possible by using new technology CCD cameras with a higher frequency of imaging and consequently, a greater accuracy in the determination of observed times. Such a camera is planned at the Bordeaux Observatory, in cooperation with the IMCCE.

\section{References}

Aksnes, K., Franklin, F., \& Magnusson, P. 1986, AJ, 92, 6

Annuaire du Bureau des Longitudes, Ephémérides astronomiques, 1997 (Masson), 1996

Arlot, J. E. 1982, A\&A, 107, 305

Arlot, J. E., Bernard, A., Bouchet, P., et al. 1982, A\&A, 111, 151

Arlot, J. E., Thuillot, W., Barrosso, J., et al. 1992, A\&AS, 92, 151

Arlot, J. E. 1996, A\&A, 314, 312

Arlot, J. E., Ruatti, C., Thuillot, W., et al. 1997, A\&AS, 125, 399

Connaissance des Temps for 1999, Bureau des Longitudes (EDP Sciences), 1998

Descamps, P. 2004, Private communication

Dourneau, G. 1982, A\&A, 112, 73

Le Campion, J. F., Montignac, G., Chauvet, F., et al. 1992, A\&A, 266, 568

Lieske 1977, A\&A, 56, 333

Lieske 1978, A\&A, 65, 83

Lieske 1980, A\&A, 82, 340

Mallama, A. 1992, Icarus, 95, 309

Thuillot, W., Arlot, J. E., Ruatti, C., et al. 2001, A\&A, 371, 343

Vasundhara, R. 1994, A\&A, 281, 565

Vasundhara, R., Arlot, J. E., Lainey, V., et al. 2003, A\&A, 410, 337 